\title{
A Correction Method for Parallel Loop Execution
}

\author{
Volodymyr Beletskyy \\ Faculty of Computer Science, Technical University of Szczecin, Zolnierska 49 st., \\ 71-210 Szczecin, Poland, \\ Bielecki@man.szczecin.pl
}

\begin{abstract}
A new method of parallel loop execution is presented. Firstly, all the loop iterations are executed in parallel. Then, the ends of pairs of dependent iterations are re-executed. The method requires no conversion of a source loop into an equivalent serial-parallel loop, involving iteration indices to be converted into new ones, that appears to be a typical requirement in the most known loop paralleling approaches such as unimodular and nonunimodular linear transformation methods. Possibilities of serial and parallel correction processes are discussed. Experimental results are considered. As follows from experiments, applying the method can be reasonable for the loops with a small fraction of dependent iterations.
\end{abstract}

\section{Introduction and related work}

Since parallel and distributed computing have become increasingly popular, it is significant to develop such compilers that would automatically translate serial programs into effective parallel code. Such code can be run on several processors of a parallel computer or on several nodes of a distributed system.

Numerous methods of loop paralleling have been proposed [1]. However, research in this field is still carried out as the problem of loop paralleling appears extremely complicated.

A new method of parallel loop execution proposed in this paper aims at maximizing the number of iterations that can be processed in parallel for the loops containing the small fraction of dependent iterations. No loop transformations need. First, all the iterations are executed in parallel. Next, dependent iterations need to be corrected. The method requires no conversion of a source loop into an equivalent serial-parallel loop, involving the source iteration indices to be converted into new ones, that appears to be a typical requirement in the most known loop paralleling methods such as unimodular and nonunimodular linear transformation approaches [2], [3], [4], [5].

The method is different from the speculative parallel execution[6] in the following: 1) dependence analysis is fulfilled before loop execution; 2) if cross-iteration dependencies exist, then only the iterations that are the ends of pairs of dependent iterations are re-executed.

The main objective of the paper is to present a concept of the correction method, possibilities of its implementation, experimental results, and future research. 


\section{Analysis of Data Dependence}

Analysis of data dependencies is considered in lots of papers [7], [8], [9]. In this section, we reproduce known knowledge that is necessary to explain a concept of the correction method.

Consider the following generic nested loop

$$
\begin{aligned}
& \text { for } I_{1}=L_{1}, U_{1} \\
& \text { for } I_{2}=L_{2}\left(I_{1}\right), U_{2}\left(I_{1}\right) \\
& \quad \ldots \quad \text { for } I_{n}=L_{n}\left(I_{1}, \ldots, I_{n-1}\right), U_{n}\left(I_{1}, \ldots, I_{n-1}\right) \\
& \quad H\left(I_{1}, \ldots, I_{n}\right) \\
& \quad \text { end for } \\
& \quad \begin{array}{l}
\text { end for }
\end{array}
\end{aligned}
$$

end for,

where $\mathrm{I}_{1}, \ldots, \mathrm{I}_{\mathrm{n}}$ are the iteration indices; $\mathrm{L}_{\mathrm{i}}$ and $\mathrm{U}_{\mathrm{i}}$ - the lower and upper loop bounds that are linear functions of the iteration indices $\mathrm{I}_{1}, \ldots, \mathrm{I}_{\mathrm{i}-1}$, implicitly a stride of one is assumed; $\mathrm{H}$ is the body of the nested loop. $\mathrm{I}=\left(\mathrm{I}_{1}, \ldots, \mathrm{I}_{\mathrm{n}}\right)^{\prime}$ is called the iteration vector.

Definition 1. The set $\mathrm{I} \subseteq \mathrm{Z}^{\mathrm{n}}$ such that

$$
\mathrm{I}=\left\{\left(\mathrm{i}_{1}, \ldots, \mathrm{i}_{\mathrm{n}}\right) \mid \mathrm{L}_{1} \leq \mathrm{i}_{1} \leq \mathrm{U}_{1}, \ldots, \mathrm{L}_{\mathrm{n}}\left(\mathrm{i}_{1}, \ldots, \mathrm{i}_{\mathrm{n}-1}\right) \leq \mathrm{i}_{\mathrm{n}} \leq \mathrm{U}_{\mathrm{n}}\left(\mathrm{i}_{1}, \ldots, \mathrm{i}_{\mathrm{n}-1}\right)\right\},
$$

is an iteration space.

Individual iterations are denoted by tuples of iteration indices.

The dependence relation between two statements constrains the order in which the statements may be executed. There are four types of data dependencies: flow, anti, output, and input dependence. The only true dependence is flow dependence. The other dependencies are the result of reusing the same location of memory and are hence called pseudo dependencies. They can be eliminated by renaming variables as well as by the techniques presented in [6], [10], [11]. From now on, we suppose that the loop has no cross-iteration anti-dependencies. Additionally, we suppose that the loop body has no statements of the form: $\mathrm{x}=\ldots \mathrm{x}$...

We often need to know all the pairs of iterations that are dependent and the precise relationship between them, such as the dependence distance vector.

Definition 2. (Dependence Distance Vector) For a pair of iterations $i=\left(i_{1}, \ldots, i_{n}\right)$ and $j=\left(j_{1}, \ldots, j_{n}\right)$ such that $j$ is flow dependent on $i$, the vector $j-i=\left(j_{1}-i_{1}, \ldots\right.$, $\left.\mathrm{j}_{\mathrm{n}}-\mathrm{i}_{\mathrm{n}}\right)^{\prime}$ is called the dependence distance vector.

From now on, we write "dependence vector" instead of "dependence distance vector". 
To find dependence vectors, equations should be built for each pair of the same named variables ID $\left(A_{1} I+B_{1}\right)$, ID $\left(A_{2} I+B_{2}\right)$ that are located in the loop body on both hand sides of assignment statements - the right and the left- or on the left-hand sides only, where $A_{1}, A_{2}$ are matrices, $B_{1}, B_{2}$ are vectors.

The equations mentioned can be written as follows:

$$
\begin{gathered}
A_{1} * i-A_{2} * j=B_{2}-B_{1}, \\
K=j-i, \\
K>0 .
\end{gathered}
$$

The solution of equations (1), if it exists, can be presented in the following form[12]:

$$
\begin{aligned}
\mathrm{K} & =\mathrm{t}_{11} * \mathrm{~V}_{1}+\mathrm{t}_{12} * \mathrm{~V}_{2}+\ldots+\mathrm{t}_{1 \mathrm{r}} * \mathrm{~V}_{\mathrm{r}}+\mathrm{V}_{0}, \\
\mathrm{i} & =\mathrm{t}_{21} * \mathrm{~W}_{1}+\mathrm{t}_{22} * \mathrm{~W}_{2}+\ldots+\mathrm{t}_{2 \mathrm{~s}} * \mathrm{~W}_{\mathrm{s}}+\mathrm{W}_{0},
\end{aligned}
$$

where: $\mathrm{V}_{0}, \mathrm{~V}_{1}, \ldots, \mathrm{V}_{\mathrm{r}}, \mathrm{W}_{0}, \mathrm{~W}_{1}, \ldots, \mathrm{W}_{\mathrm{s}}$ are vectors of dimension $\mathrm{n}$ with constant elements; $t_{1 q}, t_{2 p}, q=1,2, \ldots, r ; p=1,2, \ldots, s$ are free variables, values of which are arbitrary integer numbers, $0 \leq \mathrm{r}, \mathrm{s} \leq \mathrm{n}$; to find the boundaries of $\mathrm{t}_{1 \mathrm{q}}, \mathrm{t}_{2 \mathrm{p}}$, the loop limits should be taken into consideration.

The vector $\mathrm{i}$ describes all the iterations that form the beginnings of pairs of dependent iterations, while the vector $j=i+K$ describes the ends of those. For a pair of dependent iterations, the beginning is the iteration that is lexicographically less. To obtain correct results, all the dependent iterations have to be executed in lexicographical order.

Let us consider the example

$$
\begin{aligned}
& \text { for } \mathrm{i}_{1}=1, \mathrm{~N} \\
& \text { for } \mathrm{i}_{2}=1, \mathrm{~N} \\
& \quad \mathrm{a}\left(\mathrm{i}_{1}, \mathrm{i}_{2}\right)=\mathrm{a}\left(\mathrm{i}_{1}-2, \mathrm{i}_{2}-1\right) .
\end{aligned}
$$

Equations (1) for the loop above are as follows:

$$
\begin{aligned}
& i_{1}-j_{1}=-2, \\
& i_{2}-j_{2}=-1 .
\end{aligned}
$$

The solution to these equations is: $\mathrm{K}=(2,1)^{\prime}, \mathrm{i}=\mathrm{t}_{1}(1,0)^{\prime}+\mathrm{t}_{2}(0,1)^{\prime}$, where $\mathrm{t}_{1}$, $\mathrm{t}_{2}$ are free variables. For the vector $\mathrm{j}$, we have

$$
\mathrm{j}=\mathrm{i}+\mathrm{K}=\mathrm{t}_{1}(1,0)^{\prime}+\mathrm{t}_{2}(0,1)^{\prime}+(2,1)^{\prime} .
$$

To find the limits of $t_{1}$ and $t_{2}$, we build inequalities

$$
(1,1)^{\prime} \leq \mathrm{j} \leq(\mathrm{N}, \mathrm{N})^{\prime} \quad \text { or }
$$




$$
(1,1)^{\prime} \leq \mathrm{t}_{1}(1,0)^{\prime}+\mathrm{t}_{2}(0,1)^{\prime}+(2,1)^{\prime} \leq(\mathrm{N}, \mathrm{N})^{\prime} .
$$

From (2), we have

$$
\begin{aligned}
& 1 \leq \mathrm{t}_{1} \leq \mathrm{N}-2 \\
& 1 \leq \mathrm{t}_{2} \leq \mathrm{N}-1 .
\end{aligned}
$$

The beginnings of dependent pairs of iterations are as follows: $(1,1),(1,2), \ldots$, $(1, N),(2,1),(2,2), \ldots,(2, N), \ldots,(N, 1),(N, 2), \ldots,(N, N)$, i.e. they belong to the entire iteration space $\mathrm{I}$.

\section{Correction Method}

The correction method comprises the two steps described below.

1. All the iterations of a loop are executed in parallel applying only old values of variables (the values before the execution of iterations).

2. Given vectors $i$ and $K$, dependent iterations are determined and the correction of results for these dependent iterations is executed. The correction means a procedure of repeating the execution of the ends of all the pairs of dependent iterations in lexicographical order with regard to new values of variables.

The correction can be executed in various ways: serially or parallel, synchronously or asynchronously, statically or dynamically.

The serial correction means that only one processor fulfils the correction.

The parallel one implies that two or more processors execute the correction simultaneously.

For the synchronous correction at first, a graph representing all the dependent iterations is built. Next, the correction for the iterations of the first layer of the graph is fulfilled, then the correction is executed for the iterations of the second layer and so on, i.e. consecutive layers are scanned serially, while the iterations within each layer are corrected in parallel. If we can correct the iterations represented by the graph independently, i.e. without waiting for the finish of the execution of a particular layer of iterations, then we have the asynchronous correction.

The correction method is considered static if finding dependent iterations and planning the iterations applied for the correction take place before loop execution(at compile-time), else the method is considered as dynamic.

Let us consider the following loop:

$$
\begin{aligned}
& \text { for } i=3,6 \\
& \quad a(i)=a(i-3)+1 ;
\end{aligned}
$$

and suppose the values of vector elements before loop execution are: $a(m)=0$, $\mathrm{m}=1,2, \ldots, 6$.

When this loop is executed serially, the following values are obtained: a $(3)=1$, $\mathrm{a}(4)=1, \mathrm{a}(5)=1, \mathrm{a}(6)=2$. 
Now, let the correction method is to be applied. After the first step(parallel execution of all iterations) we have: a $(3)=1$, a $(4)=1$, a $(5)=1$, and a $(6)=1$. The vectors $K$ and $i$ for the loop considered are as follows: $K=3, i=t_{1}+2,1 \leq t_{1} \leq 4$. Here, we have the only pair of dependent iterations with its beginning in iteration 3 and its end in iteration $6(\mathrm{j}=\mathrm{i}+\mathrm{K}=3+3=6)$. It means a single correction is only required: the result of iteration 6 is calculated using the new value of iteration 3 :

$$
\mathrm{A}(6)=(3)+1=1+1=2 \text {. }
$$

The results obtained are the same as those obtained at serial loop execution.

\section{Serial Correction}

Procedures of the serial correction may be built in different ways. When a loop yields the only pair of vectors $\mathrm{i}, \mathrm{K}$, a possible procedure is as follows. From equations (1), the vectors $i, K$ are found. By means of the vector $j=i+K$, a serial loop is built that executes the correction. The loop is constructed in such a way that tuples of iteration indices yielded with the vector $\mathrm{j}$ are increased in lexicographical order. The loop, carrying out the correction, has to execute all the iterations yielded with the vector $\mathrm{j}, \mathrm{j} \in \mathrm{I}$.

Let us consider the example:

$$
\begin{aligned}
& \text { for } i=1, N \\
& \quad a(6 i-7)=a(2 i+5) ;
\end{aligned}
$$

The solution to equations (1) for the loop above is as follows:

$$
\begin{gathered}
\mathrm{i}=\mathrm{t}^{*}(1)+3, \\
\mathrm{~K}=\mathrm{t}^{*}(2), \\
\mathrm{j}=\mathrm{i}+\mathrm{K}=\mathrm{t}^{*}(1)+\mathrm{t}^{*}(2)+3,
\end{gathered}
$$

where: $1 \leq \mathrm{t} \leq\lfloor\mathrm{N} / 3-3\rfloor$.

In this case, loop, fulfilling the correction, is as follows:

$$
\begin{aligned}
& \text { for } t=1,\lfloor N / 3-3\rfloor \\
& \quad i=3 t+3 ; \\
& \quad a(6 i-7)=a(2 i+5) ;
\end{aligned}
$$

or

$$
\begin{aligned}
& t=1 ; \\
& \text { while }(i=3 t+3 \leq N) \\
& \quad a(6 i-7)=a(2 i+5) ; t=t+1 ;
\end{aligned}
$$

The example above shows that the correction is required only for every third iteration and applying the correction method might be advantageous. 


\section{Parallel Correction}

Let us now consider the case when the loop 1) yields many dependence vectors $\mathrm{K}_{1}$, $\mathrm{K}_{2}, \ldots, \mathrm{K}_{\mathrm{n}}$, but elements of them are constant; 2) $\left\{\mathrm{i}_{1}\right\}=\ldots=\left\{\mathrm{i}_{\mathrm{n}}\right\}=\mathrm{I}$, i.e. each iteration is the beginning of a pair of dependent iterations.

To implement parallel dynamic correction in such a case, the following approach can be applied. Among the vectors $\mathrm{K}_{\mathrm{i}}, \mathrm{i}=1, \ldots, \mathrm{n}$, only such vectors are selected and marked as $\mathrm{T}_{1}, \mathrm{~T}_{2}, \ldots, \mathrm{T}_{\mathrm{m}}, \mathrm{m} \leq \mathrm{n}$ that satisfy the condition:

$$
i_{1}+T_{j} \neq i_{2}+K_{i}, i=1,2, \ldots, n ; j=1,2, \ldots, m,
$$

where $i_{1}, i_{2} \in I$ and are arbitrary iterations. If for some $K_{j} \neq T_{1}, 1=1,2, \ldots, m, T_{i}$ the condition

$$
\mathrm{i}_{1}+\mathrm{K}_{\mathrm{j}}=\mathrm{i}_{2}+\mathrm{T}_{\mathrm{i}}
$$

holds, it means that all the ends of pairs of dependent iterations yielded with the vector $K_{j}$ will be held with the vector $T_{i}$ as well since for any beginning $i_{1} \in I$ of a pair of dependent iterations with the end $i_{1}+K_{j}$, an iteration $i_{2} \in I$ exists, which is the beginning of other pair of dependent iterations with the same end. To execute dependent iterations in lexicographical order, according to the approach below, vectors $K_{j} \neq T_{1}, l=1,2, \ldots, m$ must be skipped.

Let us consider the following example :

$$
\begin{aligned}
& \text { for } i=1, N \\
& a(i)=a(i-2)+a(i-3) \text {; }
\end{aligned}
$$

Here, dependence vectors are: $K_{1}=2, K_{2}=3$. Only $K_{1}$ is the independent vector since it forms all the ends of dependent iterations as the vector $\mathrm{K}_{2}$ does.

Next, all the iterations of the set $\left\{J_{1}\right\}$ are determined. The set $\left\{J_{1}\right\}$ contains the iterations of layer 1, i.e. the iterations for which the correction should be executed firstly. The set $\left\{\mathrm{J}_{1}\right\}$ can be described as follows:

$$
\left\{\mathrm{J}_{1}\right\}=\left\{\left(\left\{\mathrm{I}_{01}+\mathrm{T}_{1}\right\} \cup\left\{\mathrm{I}_{01}+\mathrm{T}_{2}\right\} \cup \ldots \cup\left\{\mathrm{I}_{01}+\mathrm{T}_{\mathrm{m}}\right\}\right) \cap \mathrm{I}\right\},
$$

where: $\left\{\mathrm{I}_{01}\right\}$ is a set of all the iterations that are the beginnings of pairs of dependent iterations, but they are not the ends of any pair of dependent iterations; $\left\{\mathrm{I}_{01}+\mathrm{T}_{\mathrm{i}}\right\}$ means that to each tuple of the set $\left\{\mathrm{I}_{01}\right\}$ the tuple $\mathrm{T}_{\mathrm{i}}$ is added; $\cup, \cap$ are the union and intersection operations on sets.

The set $\left\{\mathrm{I}_{01}\right\}$ is computed as $\left.\left\{\mathrm{I}_{01}\right\}=\mathrm{I} \backslash\left\{\mathrm{J}_{1}\right\} \cup\left\{\mathrm{J}_{2}\right\} \cup \ldots \cup\left\{\mathrm{J}_{\mathrm{n}}\right\}\right)$, where $\mathrm{J}_{\mathrm{i}}=\mathrm{i}_{\mathrm{i}}+\mathrm{K}_{\mathrm{i}}$ is the solution of equations (1) built for a particular dependence $\mathrm{i}=1,2, \ldots, \mathrm{n}$ and yielding all the ends of pairs of dependent iterations, " $"$ is the difference operation on sets. 
The sets $\left\{\mathrm{I}_{01}+\mathrm{T}_{\mathrm{i}}\right\}, \mathrm{i}=1,2, \ldots, \mathrm{m}$ contain only single representatives of identical elements. The correction is executed in parallel for all the iterations of the set $\left\{J_{1}\right\}$.

Farther, we select all the iterations of layer 2 as follows:

$$
\left\{\mathrm{J}_{2}\right\}=\left\{\left(\left\{\mathrm{J}_{1}+\mathrm{T}_{1}\right\} \cup\left\{\mathrm{J}_{1}+\mathrm{T}_{2}\right\} \cup \ldots \cup\left\{\mathrm{J}_{1}+\mathrm{T}_{\mathrm{m}}\right\}\right) \cap \mathrm{I}\right\} .
$$

All the iterations within layer 2 are executed in parallel and the procedure as above is repeated for next layer, and so on.

The pseudo code of the correction procedure above can be written as follows:

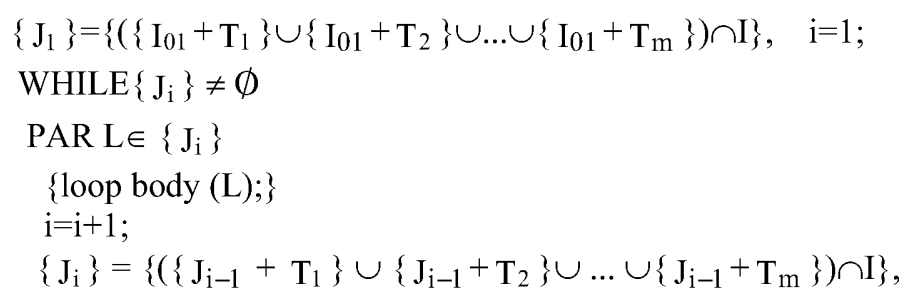

where: the instruction WHILE $\left\{\mathrm{J}_{\mathrm{i}}\right\} \neq \varnothing$ means that as long as the set $\left\{\mathrm{J}_{\mathrm{i}}\right\}$ contains elements, the loop is executed; PAR $\mathrm{L} \in\left\{\mathrm{J}_{\mathrm{i}}\right\}$ means that each iteration $\mathrm{L}$, belonging to the set $\left\{\mathrm{J}_{\mathrm{i}}\right\}$, can be executed in parallel.

The procedure above finds all the ends of pairs of dependent iterations. Iterations of each layer are independent, while for dependent iterations on consecutive layers $i$ and $i+1$, the iterations on layer $i$ are lexicographically less than those on layer $i+1$. Since layers are scanned serially at the correction, dependent iterations are executed in lexicographical order. It proves the correctness of the proposed approach.

Let us consider the loop:

$$
\begin{aligned}
& \text { for } i_{1}=1, N \\
& \text { for } i_{2}=1, N \\
& \qquad a\left(i_{1}, i_{2}\right)=a\left(i_{1}, i_{2}-1\right)+a\left(i_{1}-1, i_{2}\right)+a\left(i_{1}-1, i_{2}-1\right)
\end{aligned}
$$

The dependence vectors for the loop above are: $\mathrm{K}_{1}=(0,1)^{\prime}, \mathrm{K}_{2}=(1,0)^{\prime}, \mathrm{K}_{3}=$ $(1,1)^{\prime}$. Only the vectors $\mathrm{K}_{1}$ and $\mathrm{K}_{2}$ should be chosen for the correction. The set $\left\{\mathrm{I}_{01}\right\}$ equals $\{(1,1)\}$. The $\operatorname{set}\left\{\left\{\mathrm{I}_{01}+\mathrm{T}_{1}\right\}=\{(1,2)\}\right.$ and the set $\left\{\mathrm{I}_{01}+\mathrm{T}_{2}\right\}=\{(2,1)\}$. The loop applied to execute the correction is as follows:

$\left(\mathrm{J}_{1}\right\}=\{(1,2)\} \cup\{(2,1)\}=\{(1,2),(2,1)\}$

$\mathrm{i}=1$;

WHILE $\left\{\mathrm{J}_{\mathrm{i}}\right\} \neq \emptyset$

PAR $L \in\left\{\mathrm{J}_{\mathrm{i}}\right\}$

$$
\begin{aligned}
& a\left(i_{1}, i_{2}\right)=a\left(i_{1}, i_{2}-1\right)+a\left(i_{1}-1, i_{2}\right)+a\left(i_{1}-1, i_{2}-1\right) ; \\
& i=i+1 ; \\
& \left\{J_{i}\right\}=\left\{\left(\left\{J_{i-1}+(0,1)\right\} \cup\left\{J_{i-1}+(1,0)\right\}\right) \cap I\right\} .
\end{aligned}
$$


The number of correction layers for the loop in the last example equals to $2 \mathrm{~N}-2$. For $\mathrm{N}=3$, the iterations to be corrected on each layer are as follows:

1. $\left\{\mathrm{J}_{1}\right\}=\{(1,2),(2,1)\}$.

2. $\left\{\mathrm{J}_{2}\right\}=\left\{\mathrm{J}_{1}+(0,1)\right\} \cup\left\{\mathrm{J}_{1}+(1,0)\right\}=\{(1,2)+(0,1),(2,1)+(0,1)\} \cup\{(1,2)+(1,0),(2,1)+$ $(1,0)\}=\{(1,3),(2,2)\} \cup\{(2,2),(3,1)\}=\{(1,3),(2,2),(3,1)\}$.

3. $\left\{\mathrm{J}_{3}\right\}=\left(\left\{\mathrm{J}_{2}+(0,1)\right\} \cup\left\{\mathrm{J}_{2}+(1,0)\right\}\right) \cap \mathrm{I}=(\{(1,3)+(0,1),(2,2)+(0,1),(3,1)+(0,1)\} \cup\{(1,3)$ $+(1,0),(2,2)+(1,0),(3,1)+(1,0)\}) \cap \mathrm{I}=(\{(1,4),(2,3),(3,2)\} \cup\{(2,3),((3,2),(4,1)\}) \cap \mathrm{I}=(\{($ $1,4),(2,3),(3,2),(4,1)\}) \cap \mathrm{I}=\{(2,3),(3,2)\}$.

4. $\left\{\mathrm{J}_{4}\right\}=\left(\left\{\mathrm{J}_{3}+(0,1)\right\} \cup\left\{\mathrm{J}_{3}+(1,0)\right\}\right) \cap \mathrm{I}=(\{(2,3)+(0,1),(3,2)+(0,1)\} \cup\{(2,3)+(1,0),(3,2)$ $+(1,0)\}) \cap \mathrm{I}=(\{(2,4),(3,3)\} \cup\{(3,3),(4,2)\}) \cap \mathrm{I}=(\{(2,4),(3,3),(4,2)\}) \cap \mathrm{I}=\{(3,3)\}$.

If $\left\{\mathrm{i}_{\mathrm{i}}\right\} \neq \mathrm{I}, \mathrm{i}=1,2, \ldots, \mathrm{n}$, then the correction procedure above should be modified. After the execution of the iterations of layer $i$, from the set $\left\{J_{i}\right\}$ all the iterations that are not the beginnings of pairs of dependent iterations yielded with $\mathrm{J}_{\mathrm{i}}$ must be deleted and the iterations that are the beginnings of pairs of dependent ones given with $\mathrm{J}_{\mathrm{k}}, \mathrm{k} \neq \mathrm{i}$ must be copied to the set $\left\{\mathrm{J}_{\mathrm{k}}\right\}$.

\section{Experiments}

To evaluate the efficiency of the correction method, several experiments on Power Challenge supercomputer with three working processors and the IRIX operating system were performed. IRIX provides support for creating multithread applications by means of the Power C compiler .

The following $\mathrm{C}$ loop

$$
\begin{aligned}
& \text { for }(a 1=1 ; a 1<=n ; a 1++)\{ \\
& \mathrm{T}\left[10^{*} \mathrm{a} 1\right]=\mathrm{f}(\mathrm{T}[\mathrm{a} 1], t) ; \\
& \mathrm{T}\left[11^{*} \mathrm{a} 1\right]=\mathrm{f}(\mathrm{T}[\mathrm{a} 1], t) ; \\
& \left.\mathrm{T}\left[13^{*} \mathrm{a} 1\right]=\mathrm{f}(\mathrm{T}[\mathrm{a} 1], t) ;\right\}
\end{aligned}
$$

was executed with different implementations of the correction method, where $f(T[a 1], t)$ is a function with two parameters $T[a l]$ and $t$. The parameter $t$ defines the time of the execution of each iteration.

For this loop, there are three dependence vectors $K_{1}=9 t_{1}, K_{2}=10 t_{2}, K_{3}=12 t_{3}$ stating flow dependencies with the beginnings in the iterations $i_{1}=t_{1}, i_{2}=t_{2}$, and $i_{3}=t_{3}$ correspondingly. There are three dependence vectors $\mathrm{K}_{4}=\mathrm{t}_{4}, \mathrm{~K}_{5}=3 \mathrm{t}_{5}, \quad \mathrm{~K}_{6}=2 \mathrm{t}_{6}$ designating output dependencies with the beginnings in the iterations $i_{4}=10 t_{4}, i_{5}=10 t_{5}$, and $i_{6}=11 t_{6}$ correspondingly. Applying the correction method for the above loop is permissible since there are no anti-dependencies. The independent vectors are $\mathrm{K}_{1}, \mathrm{~K}_{4}$, and $\mathrm{K}_{6}$.

Correction procedures were implemented in accordance with the modified approach presented in Section 5. For the static correction, all the ends of pairs of dependent iterations were calculated end memorized in a container in lexicographical 
order at compile-time. At the dynamic correction, the data required were calculated at run-time. At all the cases, the results after serial loop execution and after the corrections were the same.

Figure 1 illustrates how speedup depends on the average execution time of one iteration at the execution of loop (3) with the static serial, dynamic serial, and dynamic parallel corrections.

The average execution time of one iteration is the quotient of the division of the whole time of the loop execution by the number of iterations executed. This time as well as the number of the iterations executed greatly influences speedup at parallel executing the loop on Power Challenge since creating and synchronizing threads with IRIX need some time. If this time is comparable or more than the time of the loop execution, then the efficiency of parallel applications is low enough. It is why we have investigated how speedup depends on the average execution time of one iteration and the number of iterations.

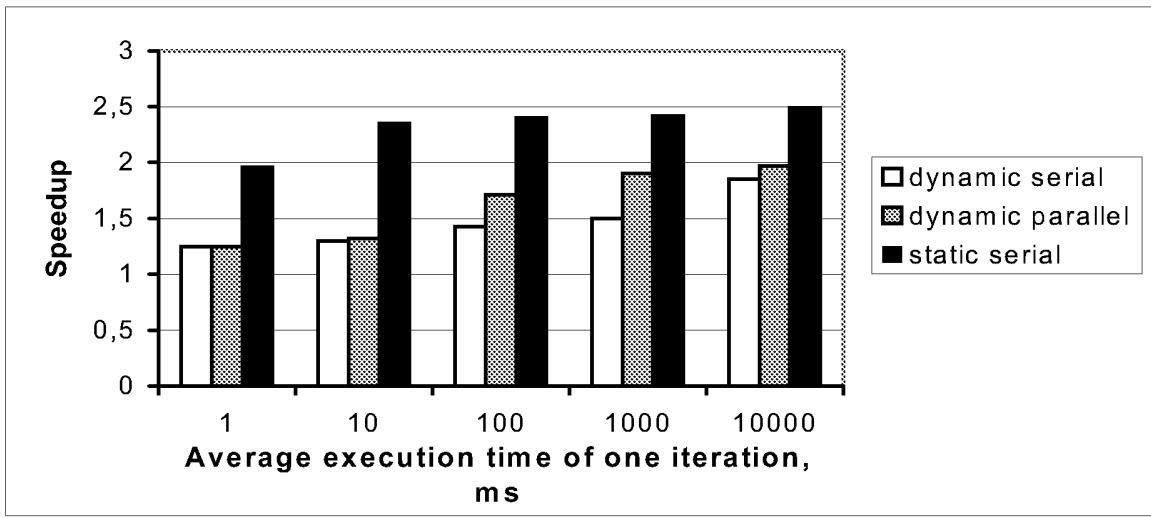

Fig.1. Speedup dependence on the average execution time of one iteration at fulfilling loop (3): 10.000 iterations, $2,6 \%$ dependent iterations

On the basis of analysis of the results received, the following conclusions can be made:

1. The top speedup was reached with the static serial correction. The explanation of this fact is that all the date necessary for the static correction are collected at compile-time, while at dynamic correction, the date are generated at run-time. But the static correction requires many memory for its implementation. All the data(dependent iterations) must be stored in memory before the loop execution.

2. As the average execution time of one iteration increases, speedup for each approach investigated increases as well since the percentage of the time required to create and synchronize parallel threads decreases.

It may be expected that if the average execution time of one iteration is large enough and the percentage of dependent iterations is small, applying the correction method can be reasonable. 


\section{Conclusion}

The concept of the correction method, enabling executing loops in parallel, was presented. The method involves two steps: the first step provides the parallel execution of all the iterations of a loop, while the second one fulfils the correction of the ends of pairs of dependent loop iterations.

Further research on the correction method needs. The main work to be done is as follows: determining a scope of effective applying the method; developing algorithms of the method implementation for different approaches: static/dynamic, serial/parallel, synchronous/asynchronous; comparing the correction method with other known loop paralleling techniques; combining the correction method with other loop paralleling approaches; program implementation of different techniques of the method; examination of the method by means of standard benchmarks and different general-purpose applications.

\section{References}

1. Bacon, D., Graham S., Sharp O.: Compiler Transformation for High-Performance Computing. Computing Surveys, 26 (4) (December, 1994) 345-420

2. Lim A.W., Lam S.: Maximizing Parallelism and Minimizing Synchronization with Affine Transformations. Parallel Computing, 24(3-4) (May, 1998) 445-475

3. Huang C.H., Sadayappan P.: Communication - Free Hyperplane Partitioning of Nested Loops. Journal of Parallel and Distributed Computing, 19(2) (October, 1993) 90-102

4. Wolfe M.: High Performance Compilers for Parallel Computing. Addison-Wesley Publishing Company (1995)

5. Ramanujam J.: Non-Singular Transformations of Nested Loops. In Supercomputing 92 (1992) 214-223

6. Rauchwerger L., Padua D.: The Privatizing Doall Test: A Run-Time Technique for Doall Loop Identification and Array Privatization. In Proc. of the 1994 International Conf. on Supercomputing (July, 1994) 33-43

7. Banerjee U.: Dependence Analysis for Supercomputing. Kluwer Academic Publishers (1988)

8. Li Z., Yew P., Zhu C.: An Efficient Data Dependence Analysis for Parallelizing Compilers. IEEE Trans. Parallel Distributed Systems, 1(1) (1990) 26-34

9. Wolfe M. Tseng Chau-Wen: The Power Test for Data Dependence. IEEE Trans. Parallel Distributed Systems, 3(5) (1992) 591-601

10. Feautrier P.: Array Expansion. Conference Proceedings on International Conference on Supercomputing, ACM (1988) 429-441

11. Brandes T.: The Importance of Direct Dependencies for Automatic Parallelization. Conference Proceedings on International Conference on Supercomputing, ACM (1988) 407- 417

12. Gregory R.I., Krishnamurthy E.V.: Methods and Applications of Error-Free Computation. Springer-Verlag (1984) 